\title{
Liposuktion beim Lipödem
}

In einer Mail vom 28.06.2019 wurde uns als Gesellschaft für Phlebologie die Möglichkeit eingeräumt, bis zum 26.07.2019 Stellung zu nehmen:

Stellungnahmerecht gemäß § 92 Absatz 7 d Satz 1 Halbsatz 1 SGB $\vee$ der einschlägigen wissenschaftlichen Fachgesellschaften, die in der AWMF organisiert sind hier: Änderung der Richtlinien Methoden Krankenhausbehandlung (KHMe-RL) und Methoden vertragsärztliche Versorgung (MVV-RL) sowie über eine Richtlinie zur Qualitätssicherung:

Liposuktion bei Lipödem im Stadium III

Erneut konnten wir feststellen, dass sich trotz der bevorstehenden Ferienzeit und der kurzen Frist binnen 2 Tagen eine Arbeitsgruppe unter der Leitung von Dr. Gabriele Färber, Hamburg, organisierte, die ein hochwertiges Papier fertigstellte, das nun dem G-BA vorliegt und am 08.08.2019 in einer Anhörung diskutiert werden wird. Über die Ergebnisse werden wir in der nächsten Ausgabe informieren. 